\title{
1 Warming impairs trophic transfer efficiency in a long-term field experiment
}

2 Diego R Barneche ${ }^{1,2,3}$, Chris J Hulatt ${ }^{4,5}$, Matteo Dossena ${ }^{4}$, Daniel Padfield ${ }^{3}$, Guy Woodward ${ }^{6}$,

3 Mark Trimmer ${ }^{4, *}$, Gabriel Yvon-Durocher ${ }^{3, *}$

$4{ }^{1}$ Australian Institute of Marine Science, Indian Ocean Marine Research Centre, Crawley, WA 6009, Australia

$5 \quad{ }^{2}$ Oceans Institute, The University of Western Australia, Crawley, WA 6009, Australia

$6 \quad{ }^{3}$ Environment and Sustainability Institute, University of Exeter, Penryn, United Kingdom, TR10 9FE

$7{ }^{4}$ School of Biological and Chemical Sciences, Queen Mary University of London, London, United Kingdom

$8{ }^{5}$ Faculty of Biosciences and Aquaculture, Nord University, Bodø, Norway

$9{ }^{6}$ Department of Life Sciences, Imperial College London, Silwood Park, Buckhurst Road, Ascot, Berkshire, SL5

10 7PY, United Kingdom

11 *e-mail: g.yvon-durocher@exeter.ac.uk or m.trimmer@qmul.ac.uk.

12 Running title: Warming reduces trophic transfer efficiency

13 Keywords: nitrogen, carbon, stoichiometry, efficiency of energy transfer, phytoplankton, zoo-

14 plankton, stable isotopes, biomass

15 Word count: 2,030 (Text), 2,983 (Methods).

16 Figures: 3; Text references: 38; Methods references: 6.

17 Extended Data Figures: 9; Extended Data Tables: 1.

18 Supplementary Figures: 13; Supplementary Tables: 2. 
In natural ecosystems, the efficiency of energy transfer from resources to consumers determines the biomass structure of food webs. As a general rule, about $10 \%$ of the energy produced in one trophic level makes it up to the next ${ }^{1-3}$. Recent theory suggests this energy transfer could be further constrained if rising temperatures increase metabolic growth $\operatorname{costs}^{4}$, although experimental confirmation in whole ecosystems is lacking. We quantified nitrogen transfer efficiency (a proxy for overall energy transfer) in freshwater plankton in artificial ponds exposed to 7 years of experimental warming. We provide the first direct experimental evidence that, relative to ambient conditions, $4^{\circ} \mathrm{C}$ of warming can decrease trophic transfer efficiency by up to $56 \%$. In addition, both phytoplankton and zooplankton biomass were lower in the warmed ponds, indicating major shifts in energy uptake, transformation and transfer ${ }^{5,6}$. These new findings reconcile observed warming-driven changes in individual-level growth costs and carbon-use efficiency across diverse taxa ${ }^{4,7-10}$ with increases in the ratio of total respiration to gross primary production at the ecosystem level ${ }^{11-13}$. Our results imply that an increasing proportion of the carbon fixed by photosynthesis will be lost to the atmosphere as the planet warms, impairing energy flux through food chains, with negative implications for larger consumers and the functioning of entire ecosystems.

Energy transfer efficiency between trophic levels has been recognised as a key determinant of how biomass is distributed in ecosystems for more than a century ${ }^{1-3,14-17}$. More efficient energy transfer across short food chains can lead to higher standing biomass of upper trophic levels: for example, inverted biomass pyramids are often seen in aquatic food webs ${ }^{18,19}$, where consumer stocks outweigh those of the smaller producers, with much higher biomass turnover rates than their animal consumers. At the other extreme, inefficient energy transfer via long food chains can explain the relatively low biomass of apex predators in other ecosystems ${ }^{16,20,21}$. Understanding how rising temperatures might alter the efficiency of energy transfer through food chains $^{22,23}$ is therefore critical for predicting how ecosystem structure and function will respond to global warming as well as for assessing impacts on commercially important apex predators, which are already under threat from a multitude of other stressors ${ }^{24}$.

47 Multiple studies suggest that elevated temperatures decrease the carbon-use efficiency or increase growth costs for individuals ${ }^{4,7-10}$ and recent theory demonstrates how higher growth 
costs could reduce energy transfer efficiency through food chains ${ }^{4}$. Although a handful of studies have indirectly inferred that rising temperatures may be linked to declines in energy transfer

51 efficiency in different systems ${ }^{22,23,25}$, direct experimental measurements have remained elusive.

52 We established an outdoor, still-water mesocosm experiment in $2005^{17}$ to address this gap, us53 ing twenty $1 \mathrm{~m}^{3}$ artificial ponds, half of which have been warmed by $4^{\circ} \mathrm{C}$ (e.g. in line with 54 IPCC Scenario A1B ${ }^{26}$ ) above ambient temperature since September 2006 (Extended Data Fig.

55 1). These ponds have been open to natural dispersal and colonisation from the regional species 56 pool for hundreds of generations and have well-established, diverse communities ${ }^{27}$, allowing us 57 to explore how warming alters ecological and evolutionary dynamics in whole ecosystems. In 582013 , after 7 years of warming, we carried out a ${ }^{15} \mathrm{~N}$ isotope tracer experiment ${ }^{28}$ to track how long-term warming had altered the trophic transfer efficiency between phytoplankton and their zooplankton consumers.

61 On the $16^{\text {th }}$ July 2013 , we added a trace amount $(980 \mu \mathrm{mol})$ of $\mathrm{K}^{15} \mathrm{NO}_{3}$ - hereafter the ${ }^{15} \mathrm{~N}$ tracer - to sixteen ponds over the course of 24 hours (Extended Data Fig. 1). The experiment was designed to trace the natural incorporation of nitrogen over time, but without perturbing the system by inducing a phytoplankton bloom due to an artificial fertilisation effect. The addition of the ${ }^{15} \mathrm{~N}$-tracer had no detectable influence on the concentration of total dissolved inorganic nitrogen, nor did it affect the daytime $\mathrm{CO}_{2}$ influx to the ponds through net primary production (see Methods, Extended Data Figs. 2,3, Supplementary Table S1, Supplementary Figs. S1-4). We quantified nitrogen transfer between phytoplankton and zooplankton as a proxy for overall energy transfer based on our finding that the biomass $\mathrm{C}: \mathrm{N}$ ratio of both plankton groups did not vary systematically within each pond during the experiment (see Methods, Supplementary Fig. $\mathrm{S} 5)$. Because the $\mathrm{C}: \mathrm{N}$ ratio within each pond remained constant while nitrogen was being assimilated, we can conclude that carbon was assimilated proportionately, supporting the assumption that the efficiency of carbon and energy transfer between trophic levels can be measured by tracing nitrogen incorporation dynamics (see Methods). The ${ }^{15} \mathrm{~N}$-tracer was quantified in each pond as ${ }^{15} \mathrm{~N}_{\%}$ (i.e. excess atom percent) relative to baseline throughout the experiment (54 days; see Methods).

Using stable isotope tracers to understand material fluxes, and how they vary with environmental gradients has a rich history in ecology ${ }^{28-31}$. We adapted a one-compartment, first-order 


$$
\chi(t)=\frac{\phi \kappa_{e} \kappa_{a}\left(e^{-\kappa_{e} t}-e^{-\kappa_{a} t}\right)}{\kappa_{a}-\kappa_{e}},
$$

83 where $\phi(\% \mathrm{~d})$ is an empirical normalisation constant. We applied a Bayesian hierarchical ap84 proach to estimate $\phi, \kappa_{a}$, and $\kappa_{e}$ for each temperature treatment (ambient vs. warmed), while 85 accounting for pond-level variation (see Methods and Extended Data Fig. 4). The model cap86 tured the ${ }^{15} \mathrm{~N}$-tracer dynamics and revealed substantial differences between treatments for both 87 phytoplankton and zooplankton (Fig. 1, Extended Data Fig. 5, Supplementary Fig. S6). Phyto88 plankton rapidly incorporated the ${ }^{15} \mathrm{~N}_{\%}$ during the first few days of the experiment (Extended 89 Data Fig. 5), whereas its uptake by the zooplankton was slower and mirrored the tracer decay 90 in the phytoplankton, highlighting the close coupling of material transfer between these trophic 91 levels. Both response curves were asymmetric (Fig. 1), with a faster approach to the peak than 92 for the decay phase ${ }^{29}$.

93 The absorption rate, $\kappa_{a}$, was unaffected by warming among the phytoplankton (ambient: median $94=0.61 ; 95 \%$ credible intervals (C.I.) $=0.35-0.89$; warmed: median $=0.62 ; 95 \%$ C.I. $=0.33-$ 95 1.03), but was elevated among the zooplankton from the warmed ponds (median $=0.17$; $95 \%$ C.I. $96=0.04-0.47)$ relative to ambient ponds (median $=0.08 ; 95 \%$ C.I. $=0.02-0.23 ;$ Fig. $2 \mathrm{a}$, Extended 97 Data Table 1). The elimination rate, $\kappa_{e}$, however, was higher in the warmed ponds for both phytoplankton (ambient: median $=0.11 ; 95 \%$ C.I. $=0.05-0.22$; warmed: median $=0.31 ; 95 \%$ C.I. $=$ $0.13-0.55$ ) and zooplankton (ambient: median $=0.09 ; 95 \%$ C.I. $=0.05-0.14$; warmed: median $100=0.14 ; 95 \%$ C.I. $=0.06-0.26$; Fig. $2 b$ ). These findings demonstrate that long-term warming 101 has fundamentally altered material flux dynamics in these plankton communities. The higher 102 rates of ${ }^{15} \mathrm{~N}$ absorption and elimination in the zooplankton, as well as higher rates of elimina103 tion in the phytoplankton are consistent with faster metabolism at elevated temperatures ${ }^{32,33}$. 104 Furthermore, the lack of a warming effect on the absorption rate, coupled with markedly faster elimination in the phytoplankton, and the substantial effects of warming on the rates of both 


$$
\varepsilon(t)=\frac{\chi(t)}{\phi \kappa_{e}\left(1-e^{-\kappa_{a} t}\right)} .
$$

116 We can then integrate equation 2 to quantify the mean efficiency of nitrogen transfer, $\bar{\varepsilon}$, over the duration of the experiment, $\tau=54$ days:

$$
\bar{\varepsilon}=\frac{\int_{t=0}^{t=\tau} \varepsilon(t) d t}{\tau} .
$$

118 For phytoplankton, $\bar{\varepsilon}$ reflects the efficiency of nitrogen uptake from the inorganic tracer pool 119 (including any recycled nitrogen through, e.g., zooplankton excretion), while for zooplankton 120 it quantifies nitrogen transfer efficiency from the phytoplankton. It is important to note that equations 1-3 constitute a phenomenological characterisation of nitrogen incorporation dynamics and transfer efficiency in that they make no attempt to mechanistically quantify the multitude of physiological (e.g. nutrient uptake, respiration, excretion, photosynthesis), ecological (e.g. predation, mortality, changes in biomass and species composition) and biogeochemical (e.g. internal nutrient recycling) processes that ultimately influence the rates of nitrogen absorption, elimination and transfer efficiency within the phytoplankton and zooplankton. Rather, any treatment effects that we observe in the model parameters $\kappa_{a}$ and $\kappa_{e}$, and the efficiency $\bar{\varepsilon}$ reflect the emergent outcome of temperature-driven shifts in some or all of these physiological, ecological and biogeochemical processes. 
130 We obtained posterior distributions of treatment-specific mean efficiencies of nitrogen trans-

131 fer, $\bar{\varepsilon}$, based on the treatment-specific Bayesian posterior distributions of $\kappa_{a}, \kappa_{e}$, and $\phi . \bar{\varepsilon}$ 132 ranged from $10-40 \%$ on average across treatments and groups (Fig. 2d), consistent with pre133 vious estimates from natural systems ${ }^{2,3}$. From the posterior draws of treatment-specific $\bar{\varepsilon}$, we 134 also obtained a distribution of the percentage decline in $\bar{\varepsilon}$ between ambient and warmed ponds, 135 which was substantially reduced in the warmed ponds for both the phytoplankton (median decline $=56.4 \%$; upper 95\% C.I. $=27.5-87.8 \%)$ and zooplankton $(38.1 \%$; upper 95\% C.I. $=3.6-$ 81.3\%) communities (Fig. 2d; Extended Data Fig. 6). A Bayesian hierarchical model, which accounted for repeated measures throughout the experiment, revealed that biomass was lower in the warmed ponds (Fig. 3) for both phytoplankton (median decline $=58.4 \%$; 95\% C.I. $=22.9$ $84.0 \%)$ and zooplankton $(65.6 \% ; 95 \%$ C.I. $=12.8-93.2 \%)$, which is consistent with reduced energy transfer efficiency altering the biomass pyramid ${ }^{17}$ (Extended Data Fig. 6, Supplementary Fig. S7).

Our findings show that the structure and functioning of the ecosystems that have emerged after 7 years of experimental warming are characterised by markedly lower trophic transfer efficiency compared with those that have assembled under ambient temperature regimes. A wide range of interrelated and non-mutually exclusive physiological, ecological and evolutionary mechanisms could provide causative explanations for these results, but such fine-grained processes cannot be disentangled in a field experiment with freely assembling ecosystems of the scale and complexity as presented in this study. Nevertheless, a number of lines of evidence provide important clues. For example, we have consistently observed that warming has shifted the phytoplankton communities towards larger species ${ }^{17,27,36}$ (Supplementary Fig. S8) that are also potentially less palatable to zooplankton consumers. Such a shift in the edibility of the phytoplankton communities could at least partially explain the lower trophic transfer efficiency in the warmed ecosystems. In contrast, the metabolic balance quantifies the overall energy balance between photosynthesis (carbon fixation) and respiration (carbon remineralisation) at the ecosystem scale and throughout this long-term experiment we have observed that warming has increased the ratio of ecosystem respiration (ER) to gross primary production (GPP) ${ }^{13,26}$ (see Supplementary Fig. S9). These results emphasise that despite shifts in taxonomic composition, the fundamental effect of warming in altering the carbon metabolism and energy balance of these 
ecosystems has remained consistent. Thus, whilst the structural elements of the ecosystems may have undergone reorganisation over time either via ecological change of the constituent taxa ${ }^{17,27}$ or via evolutionary adaptation ${ }^{37}$, the thermodynamic impacts of warming on energy metabolism seem to ultimately constrain the effects of rising temperatures on ecosystem functioning. The findings in the present manuscript - that warming has decreased the efficiency of energy transfer between trophic levels - appears to encapsulate yet another manifestation of the way in which warming has radically altered the metabolism and energy flows in these ecosystems. Together this body of evidence suggests that rising temperatures alter metabolism at the organism level which, in turn, reduces the amount of energy that can be transferred from one trophic level to the next. Ultimately this means that more of the carbon fixed by photosynthesis is respired and lost to the atmosphere as heat and $\mathrm{CO}_{2}$ with less being retained in the ecosystem. If these findings are generally applicable — and there is good reason to believe they could be $22,23,25,38$ — climate warming could cause major changes to the flux of energy and declines in the biomass of toppredators in the aquatic realm, which may impair the critical services that aquatic ecosystems deliver to society, including the provision of food from commercial fisheries.

1. Semper, K. Animal life as affected by the natural conditions of existence. 30, 488 (Appleton; Co., 1881).

2. Lindeman, R. L. The trophic-dynamic aspect of ecology. Ecology 23, 399-417 (1942).

3. Pauly, D. \& Christensen, V. Primary production required to sustain global fisheries. Nature 374, 255-257 (1995).

4. Barneche, D. R. \& Allen, A. P. The energetics of fish growth and how it constrains food-web trophic structure. Ecology Letters 21, 836-844 (2018).

5. Allen, A. P., Gillooly, J. F. \& Brown, J. H. Linking the global carbon cycle to individual metabolism. Functional Ecology 19, 202-213 (2005).

6. Yvon-Durocher, G. et al. Reconciling the temperature dependence of respiration across timescales and ecosystem types. Nature 487, 472-476 (2012).

7. Schaum, C., Buckling, A., Smirnoff, N., Studholme, D. J. \& Yvon-Durocher, G. Environmental fluctuations accelerate molecular evolution of thermal tolerance in a marine diatom. Nature 
8. Manzoni, S., Taylor, P., Richter, A., Porporato, A. \& Ågren, G. I. Environmental and sto(2012).

9. Bradford, M. A. \& Crowther, T. W. Carbon use efficiency and storage in terrestrial ecosystems. New Phytologist 199, 7-9 (2013).

10. Barneche, D. R., Jahn, M. \& Seebacher, F. Warming increases the cost of growth in a model vertebrate. Functional Ecology 33, 1256-1266 (2019).

11. Laws, E. A., Falkowski, P. G., Smith Jr., W. O., Ducklow, H. \& McCarthy, J. J. Temperature 198 (2000).

12. Davidson, E. A. \& Janssens, I. A. Temperature sensitivity of soil carbon decomposition and feedbacks to climate change. Nature 440, 165-173 (2006).

201 17. Yvon-Durocher, G., Montoya, J. M., Trimmer, M. \& Woodward, G. Warming alters the

211 size spectrum and shifts the distribution of biomass in freshwater ecosystems. Global Change 212 Biology 17, 1681-1694 (2011).

213 18. Gasol, J. M., del Giorgio, P. A. \& Duarte, C. M. Biomass distribution in marine planktonic 214 communities. Limnology and Oceanography 42, 1353-1363 (1997). 
215 19. Wang, H., Morrison, W., Singh, A. \& Weiss, H. Modeling inverted biomass pyramids and 216 refuges in ecosystems. Ecological Modelling 220, 1376-1382 (2009).

217 20. Hutchinson, G. E. Homage to Santa Rosalia or why are there so many kinds of animals? 218 The American Naturalist 93, 145-159 (1959).

219 21. Post, W. M. \& Pimm, S. L. Community assembly and food web stability. Mathematical 220 Biosciences 64, 169-192 (1983).

221 22. Šolić, M. et al. Impact of the $3^{\circ} \mathrm{C}$ temperature rise on bacterial growth and carbon transfer 222 towards higher trophic levels: Empirical models for the Adriatic Sea. Journal of Marine Systems 223 173, 81-89 (2017).

224 23. Ullah, H., Nagelkerken, I., Goldenberg, S. U. \& Fordham, D. A. Climate change could drive 225 marine food web collapse through altered trophic flows and cyanobacterial proliferation. PLOS 226 Biology 16, 1-21 (2018).

227 24. Jackson, J. B. C. et al. Historical overfishing and the recent collapse of coastal ecosystems. 228 Science 293, 629-637 (2001).

229 25. Stock, C. A. et al. Reconciling fisheries catch and ocean productivity. Proceedings of the 230 National Academy of Sciences 114, E1441-E1449 (2017).

231 26. Yvon-Durocher, G., Jones, J. I., Woodward, G., Trimmer, M. \& Montoya, J. M. Warming 232 alters the metabolic balance of ecosystems. Philosophical Transactions of the Royal Society B: 233 Biological Sciences 365, 2117-2126 (2010).

234 27. Yvon-Durocher, G. et al. Five years of experimental warming increases the biodiversity and 235 productivity of phytoplankton. PLOS Biology 13, 1-22 (2015).

236 28. Mulholland, P. J. et al. Food resources of stream macroinvertebrates determined by natural237 abundance stable $\mathrm{C}$ and $\mathrm{N}$ isotopes and $\mathrm{a}^{15} \mathrm{~N}$ tracer addition. Journal of the North American 238 Benthological Society 19, 145-157 (2000).

239 29. Crossley, Jr., D. A. \& Reichle, D. E. Analysis of transient behavior of radioisotopes in insect 240 food chains. BioScience 19, 341-343 (1969).

241 30. Mulholland, P. J. et al. Stream denitrification and total nitrate uptake rates measured using 242 a field ${ }^{15} \mathrm{n}$ tracer addition approach. Limnology and Oceanography 49, 809-820 (2004). 
243 31. López-Sepulcre, A. et al. A new method to reconstruct quantitative food webs and nutrient 244 flows from isotope tracer addition experiments. The American Naturalist online early, (2020).

245 32. Brown, J. H., Gillooly, J. F., Allen, A. P., Savage, V. M. \& West, G. B. Toward a metabolic 246 theory of ecology. Ecology 85, 1771-1789 (2004).

247 33. Gillooly, J. F., Brown, J. H., West, G. B., Savage, V. M. \& Charnov, E. L. Effects of size 248 and temperature on metabolic rate. Science 293, 2248-2251 (2001).

249 34. Gillooly, J. F. et al. The metabolic basis of whole-organism RNA and phosphorus content. 250 Proceedings of the National Academy of Sciences 102, 11923-11927 (2005).

251 35. Allen, A. P. \& Gillooly, J. F. Towards an integration of ecological stoichiometry and the 252 metabolic theory of ecology to better understand nutrient cycling. Ecology Letters 12, 369-384 253 (2009).

254 36. Padfield, D., Buckling, A., Warfield, R., Lowe, C. \& Yvon-Durocher, G. Linking phyto255 plankton community metabolism to the individual size distribution. Ecology Letters 21, 11522561161 (2018).

257 37. Schaum, C. et al. Adaptation of phytoplankton to a decade of experimental warming linked 258 to increased photosynthesis. Nature Ecology \& Evolution 1, 0094 (2017).

259 38. Rosenberg, A. A. et al. Developing new approaches to global stock status assessment and 260 fishery production potential of the seas. 1086, 175 (FAO, 2014). 
262 Fig. $1 \mid$ Temporal dynamics of the ${ }^{15}$ N-tracer, $\chi$ (excess ${ }^{15} \mathbf{N}_{\%}$ ), during the experiment.

263

264

265

a, mean predicted curves for phytoplankton and $\mathbf{b}$, zooplankton. Solid lines represent mean treatment-specific (ambient, blue vs. warmed, red) predicted curves which were obtained by fitting equation 1 to the data via a non-linear hierarchical model using a Bayesian model (see Methods). See Extended Data Fig. 5 and Supplementary Fig. S6 for pond-level mean model fits to the data and posterior predictive checks. Shaded polygons represent Bayesian $95 \%$ credible intervals which were calculated from 20,000 posterior draws. Silhouettes: CDiego Barneche.

\section{Fig. 2 | Impacts of long-term warming on the parameters that determine ${ }^{15} \mathrm{~N}$-tracer $\mathbf{d y}$ -} namics (equation 1), and the mean efficiency of nitrogen transfer (equation 3). a, absorption rate, $\kappa_{a}$, b, elimination rate, $\kappa_{e}$, $\mathbf{c}$, empirical constant, $\phi$, and $\mathbf{d}$, mean efficiency of nitrogen transfer, $\bar{\varepsilon}$. Treatment-level (ambient, blue vs. warmed, red) parameter estimates $(\mathbf{a}-\mathbf{c})$ were obtained by fitting equation 1 to the data via a non-linear hierarchical Bayesian model (see Methods). Efficiency (d) was calculated over $\tau=54$ days (duration of the experiment) based on equations 2 and 3, using the treatment-level parameter estimates. Density polygons represent Bayesian 99\% credible intervals (C.I.) which were calculated from 20,000 posterior draws. Left panels: phytoplankton; right panel: zooplankton. Silhouettes: CDiego Barneche.

Fig. 3 | Impacts of long-term warming on plankton community biomass. Mean biomass estimates were calculated from ambient (blue) and warmed (red) ponds ( $n=8$ per treatment). $y$-axis is log-scaled. Points represent mean carbon biomass for each pond calculated over the entire duration of the ${ }^{15} \mathrm{~N}$-tracer experiment (see Methods). Boxplots depict the median (mean line), as well as the first and third quartiles (lower and upper hinges). Error whiskers represent up to 1.5 times the the inter-quartile range (i.e. distance between the first and third quartiles) beyond the hinges. Shapes represent phytoplankton (top, circles) and zooplankton (squares, bottom). Silhouettes: CDiego Barneche. 


\section{Experimental set up}

288 Mesocosm pond facility. The facility was established in 2005 and consists of 20 artificial ponds 289 of about $1 \mathrm{~m}^{3}$ volume, $50 \mathrm{~cm}$ depth, sited in southern England (Freshwater Biological Associ290 ation River Laboratory, East Stoke, $2^{\circ} 10^{\prime} \mathrm{W}, 50^{\circ} 13^{\prime} \mathrm{N}$ ), designed to be broadly representative 291 of mid-latitude shallow standing waters ${ }^{17}$. Warming of $4-5^{\circ} \mathrm{C}$ above ambient began in half 292 of the ponds in September 2006 by maintaining a constant differential between thermocouples 293 in a pair of warmed and ambient ponds (Extended Data Fig. 1). The choice of $4^{\circ} \mathrm{C}$ for the

294 warmed treatment was based on the IPCC Scenario A1B for temperate regions of the Northern 295 hemisphere ${ }^{26,39}$.

296 The warming treatment has been continuously maintained until the present (May 2020). We 297 categorise the duration of the experiment as "long-term" because it encompasses enough time 298 for ecological, evolutionary and ecosystem successional dynamics to play out. Seven years (the 299 duration of the experiment at the time of the tracer additions) encompasses many hundreds to 300 thousands of generations for the planktonic organisms studied here. This means that the emer301 gent outcomes we are measuring in these systems encompass both the immediate physiological impacts of warming, as well as the changes due to local extinctions and colonisation dynamics (ecological turnover) and genetic changes in the constituent taxa as they adapt (evolutionary dynamics) to the new environmental conditions imposed by the experimental treatments ${ }^{17,27,36,37}$.

305 Taxonomic composition. The pool of species available for initial colonisation was standardised at the outset by seeding all of the ponds in December 2005 with a "common garden" inoculum of organisms from surrounding freshwater habitats. The ponds were then left open to natural colonisation and dispersal and now contain diverse multi-trophic communities that include macrophytes, macroinvertebrates ${ }^{40}$, microbes, phytoplankton and zooplankton ${ }^{17,27}$. The composition and biomass structure of these communities in the warmed and ambient treatments have

311 diverged substantially over the course of the experiment ${ }^{17,27,36}$ (see Supplementary Fig. S8).

$312{ }^{15}$ N-tracer Experiment. The tracer experiment ran from the $10^{\text {th }}$ of July 2013 to $8^{\text {th }}$ of Septem-

313 ber 2013. Before the ${ }^{15} \mathrm{~N}$-tracer experiment started a representative sample of the entire commu314 nity was collected from each of the 20 ponds. Over the course of 24 hours, starting on the $16^{\text {th }}$ of 
315 July 2013, 16 of the $1000 \mathrm{~L}$ ponds ( 8 warmed and 8 ambient) each received a total of $980 \mu \mathrm{mol}$

316 of $\mathrm{K}^{15} \mathrm{NO}_{3}$ (98 Atom\%, Sigma-Aldrich) from a $20 \mathrm{mmol} \mathrm{L}^{-1}$ stock solution. The ${ }^{15} \mathrm{~N}$-tracer was

317 added in 10 aliquots of $5 \mathrm{~mL}$ stock solution diluted in approximately $10 \mathrm{~L}$ of pond water trickled

318 over the surface of the same pond using a watering can. Each aliquot of ${ }^{15} \mathrm{~N}$-tracer was equiva-

319 lent to $\sim 0.1 \mu \mathrm{mol}{ }^{15} \mathrm{NO}_{3}{ }^{-} \mathrm{L}^{-1}$ in each pond to a total of $\sim 1 \mu \mathrm{mol}{ }^{15} \mathrm{NO}_{3}{ }^{-} \mathrm{L}^{-1}$ over 24 hours.

320 The across-time and across-pond means of dissolved inorganic nitrogen $\left(\mathrm{DIN}=\mathrm{NO}_{2}{ }^{-}+\mathrm{NO}_{3}{ }^{-}\right.$

$321+\mathrm{NH}_{4}^{+}$) was $2.87 \mu \mathrm{mol} \mathrm{L}^{-1} \pm 0.5$ (S.E.). Addition of the ${ }^{15} \mathrm{~N}$-tracer had no discernible effect on

322 the concentration of dissolved inorganic nitrogen and the daytime $\mathrm{CO}_{2}$ influx (Extended Data

323 Figs. 2,3). A further 3 of the remaining ponds were not treated, but were used as controls for

$324{ }^{15} \mathrm{~N}$ addition (Extended Data Fig. 7).

325 The water column of the ponds was sampled using a $4 \mathrm{~L}$ plastic tube open at both ends, the

326 tube was gently sunk through the water column until it reached the bottom and then closed on

327 both ends. Duplicate samples were taken from each pond so that both open water and areas

328 with macrophytes were sampled; these were then mixed and immediately taken to the on-site

329 laboratory.

330 In the laboratory, samples were sieved through a $50 \mu \mathrm{m}$ nylon mesh to isolate zooplankton. The $331<50 \mu \mathrm{m}$ fraction was filtered through a pre-ashed Whatman GF/F filter $(0.7 \mu \mathrm{m}$ nominal pore 332 size) in duplicate to isolate the phytoplankton fraction (verified by microscopy); the contents 333 of each fraction was gently rinsed with clean particulate-free water to remove any excess of ${ }^{15} \mathrm{~N}$-tracer enriched water. The $\mathrm{GF} / \mathrm{F}$ and a $30 \mathrm{~mL}$ sub-sample of water filtered at $0.7 \mu \mathrm{m}$ were immediately frozen at $-20^{\circ} \mathrm{C}$ for inorganic nutrient analysis (see below), whilst the $>50 \mu \mathrm{m}$ fraction was re-suspended in clean water and the zooplankton kept alive at room temperature to allow gut evacuation and sedimentation of the debris. After a few hours, the zooplankton were separated from water and debris and then frozen at $-20^{\circ} \mathrm{C}$.

339 Samples were collected with a decreasing frequency so that 4 sets of 16 samples of each fraction 340 were taken over the first 48 hours, starting from the addition of the first ${ }^{15} \mathrm{~N}$-tracer aliquot; then 341 one set per day was taken for the following three days; one set per week for the following month; 342 and a final set taken a month after the last sample.

343 Following the experiment, samples were analysed using a Sercon Integra 2 Isotope Ratio 
344 Mass Spectrometer (IRMS). Samples of the zooplankton fraction were quickly defrosted 345 by re-suspension in ultra-pure water and all individuals were collected under a dissection 346 microscope using forceps, placed directly in pre-weighted ultraclean tin caps $(6 \mathrm{~mm} \times 4 \mathrm{~mm}$, 347 Elemental Microanalysis, UK), dried (48 hours, $\left.60^{\circ} \mathrm{C}\right)$ and weighed on a Mettler Toledo MX5 348 precision balance. Phytoplankton samples were dried to a constant weight ( 48 hours, $60^{\circ} \mathrm{C}$ ), 349 and the dry weight of particulate matter on the filter used to calculate and standardise the 350 sample mass for IRMS. Phytoplankton sub-samples were prepared by coring the GF/F filters 351 and samples contained $14.9 \mu \mathrm{g} \mathrm{N}$ on average.

352 Samples were assembled in batches of 60 to 100 similar sample weight and each of these batches were analysed by IRMS. Two types of certified reference materials were used for this analysis: Casein $\left(\delta^{15} \mathrm{~N}+5.94 \%\right.$, $13.32 \%$ Nitrogen, $46.5 \%$ Carbon $)$ and EMA $\left(\delta^{15} \mathrm{~N}-1.57 \%\right.$, $7.46 \% \mathrm{Ni}-$ trogen, 68.35\% Carbon) (Elemental Microanalysis, UK). Casein was used for calibration of all samples. EMA was used to confirm calibration performance. Each batch of samples analysed by IRMS contained a range of urea standards covering the range of sample weights in each batch: first, 4 samples of the same reference material, then 4 samples of non-enriched urea $\delta^{15} \mathrm{~N} \approx$ $0.0 \%$ and finally 4 samples of enriched urea $\delta^{15} \mathrm{~N}=1000 \%$.

360 Data processing. For each sample, we converted the abundance of heavy nitrogen, $\delta^{15} \mathrm{~N}(\%){ }^{28}$, 361 into atom percent, ${ }^{15} \mathrm{~N}_{\%}$, as

$$
\begin{array}{r}
\delta^{15} \mathrm{~N}=\left[\frac{R_{s}}{R_{a}}-1\right] 1000 \\
{ }^{15} \mathrm{~N}_{\%}=100 \frac{\delta^{15} \mathrm{~N}+1000}{\delta^{15} \mathrm{~N}+1000+\left(\frac{1000}{R_{a}}\right)},
\end{array}
$$

362 where $R_{S}$ and $R_{a}=0.00367647$ are respectively the ${ }^{15} \mathrm{~N}:{ }^{14} \mathrm{~N}$ ratios of the sample and the atmo363 sphere. For each sample, we calculated excess ${ }^{15} \mathrm{~N}_{\%}$ over baseline abundance (i.e. $\chi$ in equation 364 1) by subtracting the natural abundance values for each taxon, in each pond, measured 7 days 365 before the addition of the $\mathrm{K}^{15} \mathrm{NO}_{3}$ tracer.

$366 \mathrm{CO}_{2}$ and dissolved inorganic nitrogen. Daytime $\mathrm{CO}_{2}$ influx $\left(\mu \mathrm{mol} \mathrm{m} \mathrm{m}^{-1}\right)$ was measured 


$$
\begin{array}{r}
m(t)=\frac{m_{0} \kappa_{a}\left(e^{-\kappa_{e} t}-e^{-\kappa_{a} t}\right)}{\kappa_{a}-\kappa_{e}} \\
c(t)=\frac{m_{0} \kappa_{e} \kappa_{a}\left(e^{-\kappa_{e} t}-e^{-\kappa_{a} t}\right)}{\theta\left(\kappa_{a}-\kappa_{e}\right)},
\end{array}
$$

379 where $m_{0}$ is the mass of tracer added to the ponds on time $t=0$, and $\theta=v \kappa_{e}$ is the clearance 380 rate (volume / time), with $v$ representing the compartment biovolume. It follows from this type of model that the mass of the ${ }^{15} \mathrm{~N}$-tracer, $m_{0}$, will be absorbed at an exponential rate; thus, we can calculate the mass of the ${ }^{15} \mathrm{~N}$-tracer that was absorbed into the compartment since time 0 as $m_{a}(t)=m_{0}\left(1-e^{-\kappa_{a} t}\right)$, such that $m_{a}(t) \equiv m_{0}$ when $t$ is large - this assumes that $m_{0}$ is $100 \%$ absorbable.

385 It is important to emphasise that equation 1 is a phenomenological adaptation of equation 5, tailored to describe the dynamics of excess ${ }^{15} \mathrm{~N}_{\%}, \chi$, observed in our experiment. As noted in the main text, parameters $\kappa_{a}$ and $\kappa_{e}$ emerge from multiple potential physiological and ecological processes that cannot be disentangled with this type of experiment. Moreover, a clearance rate is impractical to determine because $v$ represents the (unknown) biovolume of phytoplankton and 
zooplankton. Therefore, in equation 1 , we collapsed the ratio $m_{0} / \theta$ into the empirical constant, $\phi$, noting that its units $(\% \mathrm{~d})$ are different because $\chi(t)$ in equation 1 is expressed as an excess atom percent rather than concentration or mass as in equation 5 above. It also follows that the product $\phi \kappa_{e}$ is analogous to the ratio $m_{0} / v$. We empirically demonstrate in the online Supplementary information how we can quantify the efficiency of ${ }^{15} \mathrm{~N}$ transfer at time $t$ (i.e. equation 2) using three equivalent expressions.

Model fitting. We adopted a hierarchical model based on equation 1, which was implemented in a Bayesian framework using the R package $\operatorname{rstan}^{42}$ version 2.21.3 to determine posterior distributions and associated 95\% credible intervals (C.I.) for the fitted parameters (Extended Data Fig. 4). We fitted two models, one for each group (i.e. phyoplankton and zooplankton). Parameters $\kappa_{a}, \kappa_{e}$ and $\phi$ were sampled from $m$ treatment-level distributions (warmed vs. ambient), and additional uncertainty within each of these distributions was estimated at the pond level, $j$ $=\{1-8\}$, within each treatment (i.e. 8 ponds per treatment; see Extended Data Fig. 4). A series of transformations were adopted to improve convergence and run speed; (1) $\kappa_{a}$ was estimated on the natural $\log$ scale, such that pond-level $\kappa_{a[m, j]}=\exp \left(\overline{\ln \kappa_{a[m]}}+\ln \Delta \kappa_{a[m, j]}\right) ;(2)$ to ensure the constraint $\kappa_{e}<1, \kappa_{e}$ was estimated using a logit transformation, $\kappa_{e}^{\prime}$, such that pond-level $\kappa_{e[m, j]}=1 /\left(1+\exp \left(-\left(\overline{\kappa_{e[m]}^{\prime}}+\Delta \kappa_{e[m, j]}^{\prime}\right)\right)\right) ;(3)$ convergence was achieved by enforcing the constraint $\phi<1 / \kappa_{e}$ (i.e. assuming $\theta \ll v$ and $\kappa_{e}<1$ in equation 5), hence $\phi$ was estimated using a logit transformation, $\phi^{\prime}$; (4) for phytoplankton, $\phi$ was calculated from $\phi^{\prime}$ and transformed to the natural $\log$ scale, such that pond-level $\phi_{[m, j]}=\exp \left(\overline{\ln \phi_{[m]}}+\Delta \ln \phi_{[m, j]}\right) ;(5)$ for zooplankton, pond-level $\phi_{[m, j]}=\left(1 / \kappa_{e[m, j]}\right) /\left(1+\exp \left(-\left(\overline{\phi^{\prime}{ }_{[m]}}+\Delta \phi_{[m, j]}^{\prime}\right)\right)\right)$.

We used treatment and group-agnostic, weakly informative priors (Extended Data Fig. 4; Supplementary Fig. S10) for all parameters. For the treatment-level means $\overline{\ln \kappa_{a m}}, \overline{\kappa_{e m}^{\prime}}$ and $\overline{\phi^{\prime}{ }_{m}}$, we used $\mathscr{N}(0,1)$. Pond-level hierarchical deviations from treatment-level means $\left(\ln \Delta \kappa_{a[m, j]}\right.$, $\left.\Delta \kappa_{e[m, j]}^{\prime}, \Delta \ln \phi_{[m, j]}, \Delta \phi_{[m, j]}^{\prime}\right)$ were assumed to be normally distributed with means of 0 , thus the treatment-level means $\left(\overline{\ln \kappa_{a}}, \overline{\kappa_{e}^{\prime}}, \overline{\ln \phi}, \overline{\phi^{\prime}}\right)$ are among-pond means: $\ln \Delta \kappa_{a[m, j]} \sim \mathscr{N}\left(0, \sigma_{\ln \Delta \kappa_{a}}\right)$, $\Delta \kappa_{e[m, j]}^{\prime} \sim \mathscr{N}\left(0, \sigma_{\Delta \kappa_{e}^{\prime}}\right), \Delta \ln \phi_{[m, j]} \sim \mathscr{N}\left(0, \sigma_{\Delta \ln \phi}\right), \Delta \phi_{[m, j]}^{\prime} \sim \mathscr{N}\left(0, \sigma_{\Delta \phi^{\prime}}\right)$. For the hyper priors $\sigma_{\ln \Delta \kappa_{a}}, \sigma_{\Delta \kappa_{e}^{\prime}}, \sigma_{\Delta \ln \phi}$ and $\sigma_{\Delta \phi^{\prime}}$, we used $\Gamma(2,0.1)$.

8 The posterior distributions of model parameters (Extended Data Table 1) were estimated using Markov chain Monte Carlo (MCMC) methods by constructing four chains of 30,000 steps each, 
with each starting at a distinct point drawn at random from the prior distributions. Most of these iterations $(25,000)$ were used as a warm-up, so a total of 20,000 steps were retained to estimate posterior distributions (i.e. $4 \times(30,000-25,000)=20,000)$. All four independent chains reached convergence, i.e. the Gelman-Rubin statistic ${ }^{43}, \widehat{R}$, was 1 .

\section{Linking nitrogen to carbon and energy transfer efficiency}

We used a ${ }^{15} \mathrm{~N}$-tracer to quantify material transfer between trophic levels in the plankton food web, assuming that our measurements of the efficiency of nitrogen transfer also reflect carbon and energy transfer between trophic levels. To verify this assumption, we first tested whether there were any within-pond temporal changes in $\mathrm{C}: \mathrm{N}$ ratio in the ambient and warmed ponds by fitting a Bayesian hierarchical linear model to each group (phytoplankton and zooplankton). If over the duration of the 54 day experiment, which encompassed several turnovers in the shortlived phyto- and zooplankton communities, the $\mathrm{C}: \mathrm{N}$ ratio remained constant while nitrogen was being assimilated, then we can conclude that carbon was being assimilated proportionately. We included a fixed-effect interaction between time (continuous: day) and treatment (categorical: ambient vs. warmed), and a pond-level random effect to account for repeated measures throughout the experiment. C:N ratios were calculated based on moles of carbon and nitrogen in each sample on each day. A time slope, $\beta_{t}$, that is indistinguishable from 0 would be considered as evidence of no change in $\mathrm{C}: \mathrm{N}$ ratio over the ${ }^{15} \mathrm{~N}$-tracer experiment, and our results support this assumption: $\mathrm{C}: \mathrm{N}$ ratio did not change over time for both phytoplankton ( $\beta_{t}$ for ambient treatment: -0.02 ; Bayesian 95\% C.I. $=-0.08-0.03 ; \beta_{t}$ for warmed treatment: $-0.03 ; 95 \%$ C.I. $=-0.08-0.03$ ) or zooplankton $\left(\beta_{t}\right.$ for ambient treatment: $-0.01 ; 95 \%$ C.I. $=-0.04-0.02 ; \beta_{t}$ for warmed treatment: $0.02 ; 95 \%$ C.I. $=-0.01-0.04)$. These results reflect the fact that carbon biomass differences between treatments (Fig. 3) mirror those of nitrogen biomass (Extended Data Fig. 8). Pond-level C:N ratio means are shown in Extended Data Fig. 9. Together, these lines of evidence support our key assumption that the assimilation and trophic transfer of nitrogen can be used as a direct proxy for the assimilation and transfer of carbon and energy.

We then tested whether there was a decline in plankton carbon biomass between ambient and warmed treatments (Fig. 3 in the main text) that would be consistent with a decline in the efficiency of energy transfer, by fitting a Bayesian hierarchical linear model to the biomass estimates 
449 for each group. We included treatment (ambient vs. warmed) as a fixed effect, and pond as a

450 random effect to account for repeated measures throughout the experiment. Biomass data were

451 normalised by applying a natural-logarithm transformation. One of the samples presented an un-

452 usually high carbon biomass of phytoplankton (10-fold higher than the mean; Fig. 3) and was

453 therefore removed from the analysis. We used the posterior distribution of estimated parameters,

$454 \beta_{w}$ (mean carbon biomass from warmed treatments) and $\beta_{a}$ (mean carbon biomass from ambi-

455 ent treatments), to calculate a posterior distribution of between-treatment percentage decline for

456 both groups: $\left(1-\left(\beta_{w} / \beta_{a}\right)\right) \times 100$. These distributions were overlaid on the percentage-decline

457 posterior distributions obtained for the efficiency of nitrogen transfer described in the main text.

458 For phytoplankton, the posterior distribution of carbon biomass \% decline is virtually identical

459 to that of the percentage-decline in the efficiency of nitrogen transfer. For zooplankton, there

460 were subtle differences in means although the distributions overlapped over most of the range

461 (Extended Data Fig. 6). These data provide clear evidence of a decline in plankton biomass

462 between ambient and warmed treatments that is consistent with an impaired energy transfer

463 efficiency.

464 Models were fitted using the R package $b_{r m s}{ }^{44}$ version 2.14.4. Priors were uninformative (brms

465 default), and fitting specifications (number of chains, warm-up period) and convergence crite-

466 rion are the same as described above for equation 1.

\section{Before-after analyses}

468 We ran multiple before-after analyses to test whether the addition of the tracer had a discernible

469 effect on the dynamics of nitrogen incorporation in the plankton, and whether that exhibited

470 any interactions with the temperature treatment. Multiple dissolved inorganic nitrogen species

$471\left(\mathrm{NO}_{2}{ }^{-}, \mathrm{NO}_{3}{ }^{-}, \mathrm{NH}_{4}{ }^{+}\right.$; Extended Data Fig. 2) as well as daytime $\mathrm{CO}_{2}$ influx (Extended Data

472 Fig. 3) were used as response variables, each in a separate model. For dissolved inorganic nitro-

473 gen species, measurements were compared between treatments and time periods $\left(10^{\text {th }}-15^{\text {th }}\right.$ July

$4742013=$ "before"; $17^{\text {th }}$ July-06 ${ }^{\text {th }}$ August = "after") which were designated relative to the addition

475 of the ${ }^{15} \mathrm{~N}$-tracer on the $16^{\text {th }}$ of July 2013 . For daytime $\mathrm{CO}_{2}$ influx, measurements were taken

476 throughout the week "before" $\left(9^{\text {th }}-15^{\text {th }}\right)$, and "after" $\left(17^{\text {th }}-23^{\text {th }}\right)$ the addition of the ${ }^{15} \mathrm{~N}$-tracer.

477 We fitted the before-after model as an interaction between period (before, after) and treatment 
(warm, ambient) using a Bayesian hierarchical approach, with pond added as an intercept-level random effect. The before-after model was fitted using the $\mathrm{R}$ package brms $^{44}$ version 2.14.4. Priors were uninformative (brms default), and fitting specifications (number of chains, warmup period) and convergence criterion are the same as described above for equation 1 . The test revealed no discernible interaction between treatment and period for any of the dissolved inorganic nitrogen species nor daytime $\mathrm{CO}_{2}$ influx (Extended Data Figs. 2,3; Supplementary Table S1).

\section{Main model residual analysis}

We tested whether other physico-chemical properties besides temperature could be affecting the variability in the tracer incorporation dynamics. To do so, we first calculated the mean posterior observation-level residuals from our main model for each taxonomic group (i.e. phytoplankton and zooplankton; Fig. 1; Extended Data Fig. 5; Extended Data Table 1). Then, for each group separately, we employed a Bayesian hierarchical model to investigate the relationship between the residuals from the original model and the dissolved inorganic nitrogen species $\left(\mathrm{DIN}=\mathrm{NO}_{2}{ }^{-}\right.$, $\mathrm{NO}_{3}{ }^{-}, \mathrm{NH}_{4}{ }^{+}$) in the ponds. The model accounted for the repeated measurements at the pond level as hierarchical effects both on the intercept and slopes of DIN species. The model was fitted using the $\mathrm{R}$ package $b r m s^{44}$ version 2.14.4. Priors were uninformative (brms default), and fitting specifications (number of chains, warm-up period) and convergence criterion are the same as described above for equation 1. Results indicate that the DIN species could not explain any systematic variation in our main model residuals (Supplementary Table S2; Supplementary Figs. S11-13). That is, the main statistical analysis in our manuscript identifies a strong, main effect of temperature that is not improved by adding the effect of inorganic nutrients.

\section{Data and Code Availability}

All data and R code (data manipulation, analyses, figures and tables) can be downloaded from a GitHub repository (https://github.com/dbarneche/nature20200508666). When using the data or code from this project, please cite it as "Barneche DR, Hulatt CJ, Dossena M, Padfield D, Woodward G, Trimmer M, Yvon-Durocher G (2021) dbarneche/nature20200508666: Accepted version of paper data and code of manuscript: Warming impairs trophic transfer efficiency in a long-term field experiment (Nature). Zenodo. doi: 10.5281/zenodo.4468371" 
39. IPCC. in Contribution of Working Group I to the fourth assessment report of the Intergovernmental Panel on Climate Change (eds. Parry, M. L., Canziani, O. F., Palutikof, J. P., van der Linden, P. J. \& E, H. C.) 7-22 (Cambridge University Press, 2007).

40. Dossena, M. et al. Warming alters community size structure and ecosystem functioning. Proceedings of the Royal Society B: Biological Sciences 279, 3011-3019 (2012).

41. Lansdown, K. et al. Importance and controls of anaerobic ammonium oxidation influenced by riverbed geology. Nature Geoscience 9, 357-360 (2016).

42. Stan Development Team. RStan: The R interface to Stan. (2020).

43. Gelman, A. \& Rubin, D. B. Inference from iterative simulation using multiple sequences. Statistical Science 7, 457-472 (1992).

44. Bürkner, P. Advanced Bayesian multilevel modeling with the R package brms. The R Journal 10, 395-411 (2018).

Acknowledgements We thank JH Brown and four anonymous referees for constructive suggestions that greatly improved the manuscript. We thank for feedback on an earlier version of this manuscript; TJ McKinley for providing feedback on the statistical analysis. Funding: AXA Research Fund (MD), the Natural Environment Research Council to MT and GW (NE/H022511/1). GY-D was supported by an European Research Council (ERC) grant (ERC StG 677278 TEMPDEP).

Author contributions G.Y.D. and M.T. conceived the study; C.J.H. and M.D. collected the data and did the stable isotope analysis; D.P. collected the phytoplankton community data from 2016; D.R.B., G.Y.D., and M.T. conducted the statistical analyses; D.R.B. and G.Y.D. wrote the first version of the manuscript and all authors contributed substantially to revisions.

Competing interests The authors declare no competing interests.

\section{Additional information}

Supplementary information is available for this paper.

Correspondence and requests for materials should be addressed to G.Y.D. and M.T. 


\section{Extended Data}

534 Extended Data Table 1 | Parameter estimates from equation 1, which characterises the tem535 poral dynamics of the ${ }^{15} \mathbf{N}$-tracer. Mean parameter estimates, $95 \%$ credible intervals (lower 536 and upper bound), effective sample size, and Gelman-Rubin statistic ${ }^{43}, \widehat{R}$, were obtained using 537 a Bayesian hierarchical model. Parameter notation and model fitting approach are described 538 in subsection Model framework of Methods. "amb" = ambient temperature; "war" = warmed $539\left(+4^{\circ} \mathrm{C}\right)$ relative to ambient temperature. Overall treatment- and group-level model fits are visu540 ally depicted in Fig. 1; pond-level model fits are depicted in Extended Data Fig. 5. 
541 Extended Data Figure 1 | Schematic of experimental pond set-up and ${ }^{15} \mathbf{N}$-tracer measure-

542 ments. a, Twenty artificial ponds, with 10 warmed (red) by $4^{\circ} \mathrm{C}$ above (since September 2006)

54310 ambient (blue) ponds, were paired in a randomized block design. b, Ponds were controlled

544 via two temperature sensors, a heating element (HE) a thermostat (T-stat) and a solid-state relay

545 (SSR). c, Timeline of experimental measurements, including quantification of baseline ${ }^{15} \mathrm{~N}_{\%}$ of

546 phytoplankton and zooplankton before the addition of the $\mathrm{K}^{15} \mathrm{NO}_{3}$ tracer, followed by continu-

547 ous sampling of excess ${ }^{15} \mathrm{~N}_{\%}$ relative to baseline on each pond. d, Dissolved oxygen saturation

548 and $\mathrm{pH}$ did not change before and after the addition of the tracer (see ref. ${ }^{13}$ for measurement

549 details). Symbols represent treatments: ambient (blue triangles) and warmed (red inverted tri-

550 angles). Silhouettes: CDiego Barneche.

551 Extended Data Figure 2 | Concentration of dissolved inorganic nitrogen species in the 552 ponds before and after the addition of the ${ }^{15} \mathrm{~N}$-tracer on the $16^{\text {th }}$ July 2013. Addition of 553 the ${ }^{15} \mathrm{~N}$-tracer had no discernible effect on the natural concentration of dissolved inorganic ni554 trogen in the ponds (Supplementary Table S1). Points are treatment-level means, error bars are $55595 \%$ confidence intervals. Dashed line marks $16^{\text {th }}$ July.

556

Extended Data Figure 3 | Daytime $\mathrm{CO}_{2}$ influx before and after the addition of the ${ }^{15} \mathrm{~N}$ tracer on the $16^{\text {th }}$ July 2013. Each point represents an individual measurement within a pond ( $n=56$ measurements per treatment per period; as described in detail in ref. ${ }^{13}$ ). Colours refer to ambient (blue triangles) and warmed (red inverted triangles) ponds. "Before" measurements were taken daily throughout the week leading to the addition of the ${ }^{15} \mathrm{~N}$-tracer on the $16^{\text {th }}$ of July $2013\left(9^{\text {th }}-15^{\text {th }}\right)$, whereas “After" measurements were taken daily throughout the week following the addition of the tracer $\left(17^{\text {th }}-23^{\text {th }}\right)$. Boxplots depict the median (mean line), as well as the first and third quartiles (lower and upper hinges). Error whiskers represent up to 1.5 times the the inter-quartile range (i.e. distance between the first and third quartiles) beyond the hinges. Outliers were removed from the plot for visualisation purposes only. A before-after analysis (see Supplementary Table S1) revealed no substantial changes in daytime $\mathrm{CO}_{2}$ influx and net primary production due to the addition of the ${ }^{15} \mathrm{~N}$-tracer.

Extended Data Figure 4 | Hierarchical model structure for the fitting of equation 1. Data, processes and parameters are explicitly identified, with equation 1 parameters $\phi, \kappa_{a}$ and $\kappa_{e}$ being fitted at the treatment level with pond-level deviations. Phytoplankton and zooplankton 
silhouettes depict whether a certain transformation or prior was used for either group or both (see Methods). Silhouettes: CDiego Barneche.

Extended Data Figure 5 | Temporal dynamics of the ${ }^{15}$-tracer, $\chi$ (excess $\left.{ }^{15} \mathbf{N}_{\%}\right)$, in phytoplankton and zooplankton during the experiment. Dashed lines represent mean pond-level predictions which were obtained by fitting the data to equation 1 via a non-linear hierarchical Bayesian model (see Methods). Shaded polygons represent Bayesian 95\% credible intervals which were calculated from 20,000 posterior draws. Note the sharp increase in the $\chi(t)$ in the first few days of the experiment, particularly when compared to baseline ${ }^{15} \mathrm{~N}_{\%}$ in the control ponds (Extended Data Fig. 7).

\section{Extended Data Figure 6 | Posterior distributions of percentage decline in carbon biomass} $\left(\mu \mathrm{g} \mathrm{C} \mathrm{L}^{-1}\right)$ and efficiency of nitrogen transfer due to long-term warming. Distributions were calculated using 20,000 posterior draws which were estimated via Bayesian hierarchical linear models (see Methods). Positive and negative values represent percentage decline and increase respectively. The strong overlap between distributions corroborates the assumption that mean nitrogen transfer efficiency, $\bar{\varepsilon}$, as calculated from the ${ }^{15} \mathrm{~N}$-tracer dynamics (equation 3 ), reflects the efficiency of carbon and hence energy transfer. Silhouettes: CDiego Barneche.

\section{Extended Data Figure 7 | Measurements of ${ }^{15} \mathbf{N}_{\%}$ (atom percent) in three untreated control} ponds. Green circles represent phytoplankton ( $n=5$ per pond), whereas brown squares represent zooplankton ( $n=3-5$ per pond). These results are expected given that no tracer was added. The $y$-axis was kept fixed in order to compare the magnitude of change between treatments (see Extended Data Fig. 5) and controls. Refer to the Methods section for further explanations about how the data were collected.

\section{Extended Data Figure 8 | Impacts of long-term warming on mean nitrogen biomass. Mean} biomass nitrogen estimates were calculated from ambient and warmed ponds. Points represent means calculated for the entire duration of the ${ }^{15} \mathrm{~N}$-tracer experiment ( $n=8$ per treatment). Boxplots depict the median (mean line), as well as the first and third quartiles (lower and upper hinges). Error whiskers represent up to 1.5 times the the inter-quartile range (i.e. distance between the first and third quartiles) beyond the hinges. Shapes represent phytoplankton (top, circles) and zooplankton (squares, bottom). Silhouettes: CDiego Barneche. 
600 Extended Data Figure 9 | Impacts of long-term warming on C:N ratios. Mean C:N ratios

601 were calculated from ambient and warmed ponds. Points represent means calculated for the 602 entire duration of the experiment ( $n=8$ per treatment). Boxplots depict the median (mean line), 603 as well as the first and third quartiles (lower and upper hinges). Error whiskers represent up to 6041.5 times the the inter-quartile range (i.e. distance between the first and third quartiles) beyond 605 the hinges. Shapes represent phytoplankton (top, circles) and zooplankton (squares, bottom). 606 Silhouettes: CDiego Barneche. 


\section{Ambient Warmed}

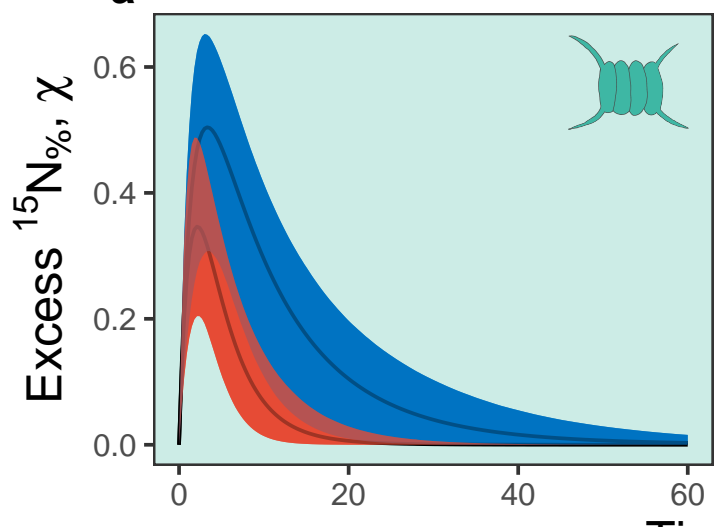

b

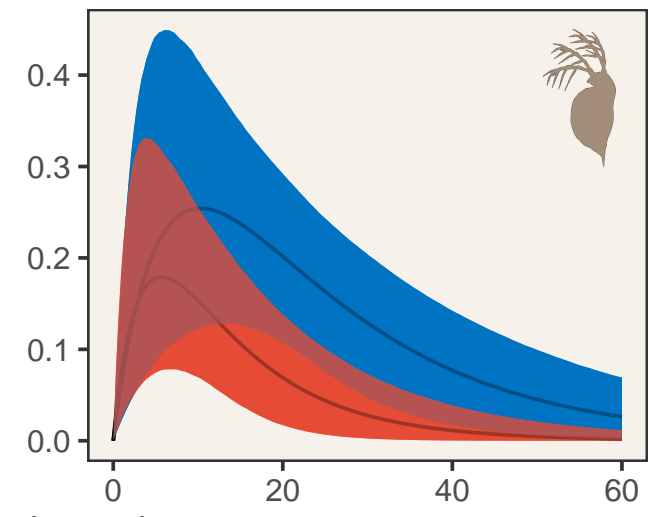

Time (days) 
a

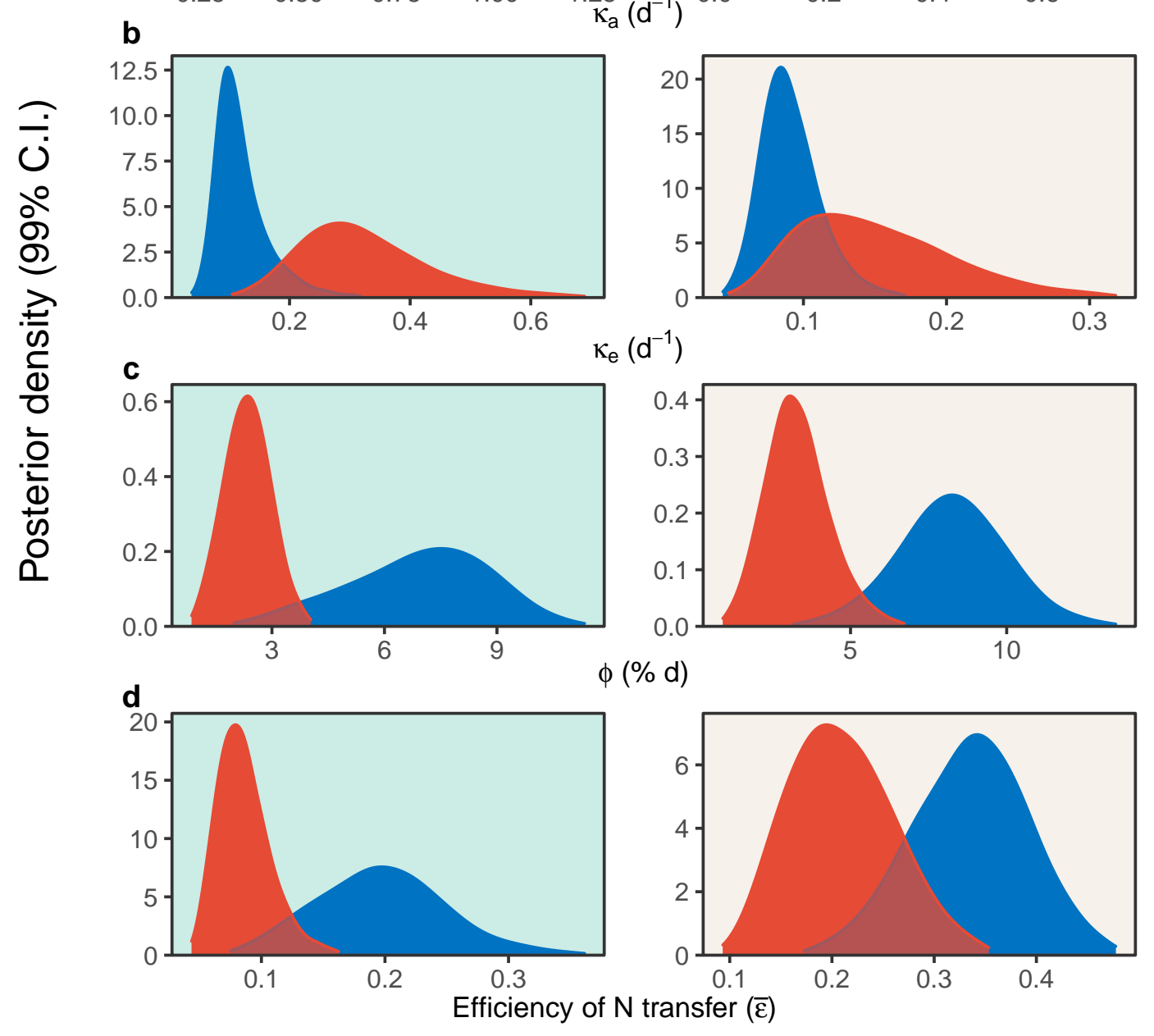




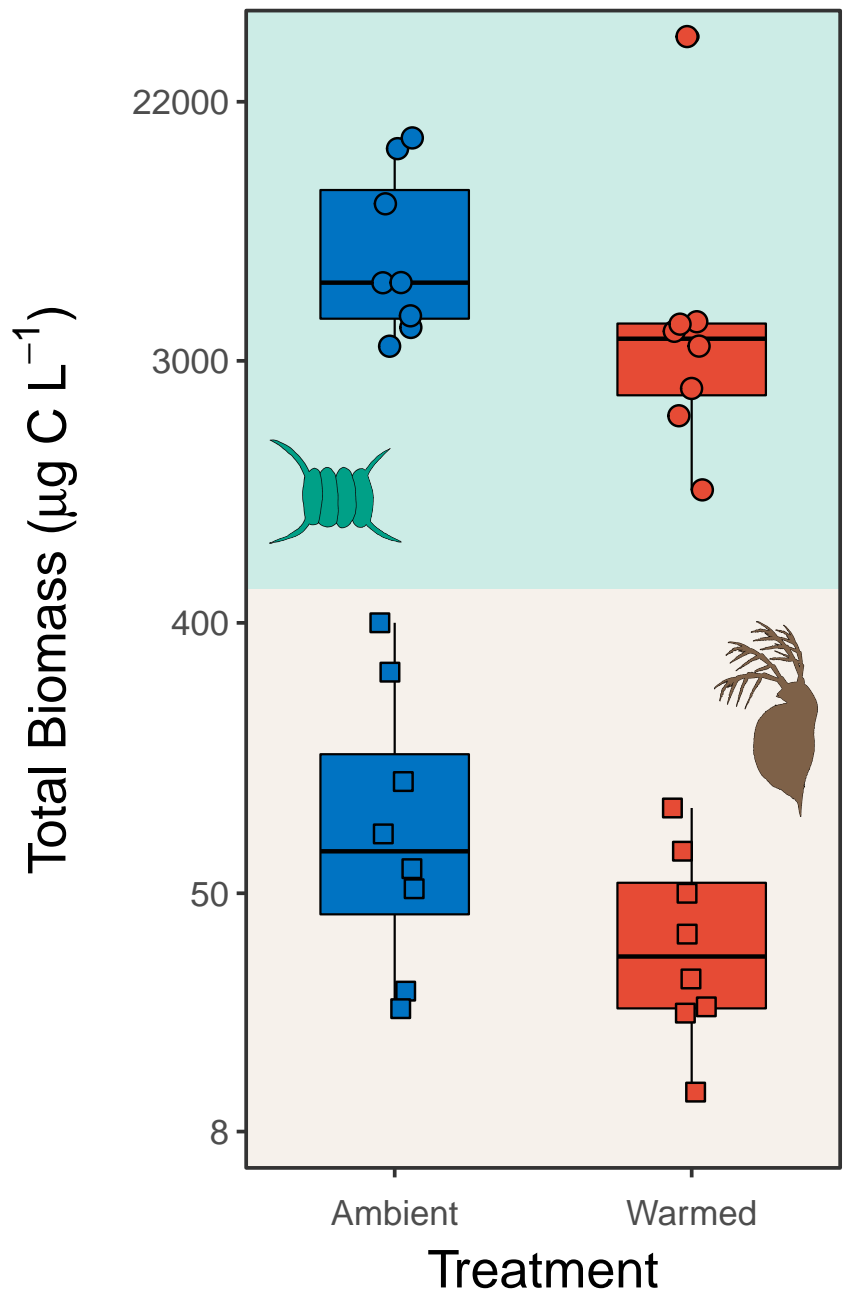

